\title{
Variation of the Milk Antibody Response to Paratuberculosis in Naturally Infected Dairy Cows
}

\author{
S. S. Nielsen*, Y. T. Gröhn, † and C. Enevoldsen‡ \\ *Department of Animal Science and Animal Health, \\ The Royal Veterinary and Agricultural University, \\ Grønnegårdsvej 8, DK-1870 Frederiksberg C, Denmark \\ †Department of Population Medicine and Diagnostic Sciences, \\ College of Veterinary Medicine, \\ Cornell University, New York 14853, USA \\ ‡Epivetko Aps, Tornager 2, DK-7600 Struer, Denmark
}

\section{ABSTRACT}

A longitudinal study was performed to determine the course of the milk antibody response in cows presumably infected with Mycobacterium avium subsp. paratuberculosis. Milk samples were collected repeatedly (1 to 10 times) from all lactating cows in seven Danish dairy herds. A total of 4289 observations from 812 cows was analyzed after exclusion of samples collected after 280 days in milk (DIM). The level of antibodies in the milk samples was assessed using an indirect ELISA.

A piece-wise linear random coefficient regression model was specified. The model controlled for the effect of herd, breed, laboratory effects, and age at first calving to estimate parity-specific antibody responses in relation to DIM. Separate antibody profiles were estimated for fecal culture-positive and fecal culture-negative cows. The resulting population average models showed higher antibody levels for fecal culture-positive cows and higher antibody levels with increasing parity. On average, the antibody response was high at the beginning and end of lactation. However, evaluating the cows individually indicated that most cows actually had quite stable ELISA levels throughout lactation, with some cows having higher levels than others. Thus, two criteria seem applicable to assess whether a cow is infected: stability and ELISA level. The random coefficients for each cow were highly significant. Thus, the study suggests that all cows can be classified into one of the four categories by combining the cow-level ELISA characteristics "stability" and "level" as an aid in the diagnosis of paratuberculosis and thereby substantially increasing the sensitivity of the ELISA.

(Key words: ELISA, paratuberculosis, immune dynamics)

Received May 7, 2001.

Accepted March 20, 2002.

Corresponding author: S. S. Nielsen; e-mail: ssn@kvl.dk.

\begin{abstract}
Abbreviation key: $\mathbf{F C}=$ fecal culture, $\mathbf{O D}=$ optical density.
\end{abstract}

\section{INTRODUCTION}

Paratuberculosis in cattle is a chronic mycobacterial infection (Chiodini et al., 1984). The disease is fairly prevalent in traditional dairy countries (Muskens et al., 2000; Nielsen et al., 2000; NAHMS, 1997), and the economic importance of the disease is high when issues such as zoonotic risk, international trade, and welfare are considered, rather than just focusing on traditional animal productivity measures (Wells et al., 1998). However, the chronicity of the infection complicates diagnostic issues important for control of the infection.

Infection status can be based on finding the causative organism, Mycobacterium avium subsp. paratuberculosis (or a part of it), or detection of an immune response. Finding the organism using premortem diagnostic tests usually implies that the animal is shedding the mycobacterium. Infected animals shedding the bacteria are a minority of the total number of infected animals (Whitlock and Buergelt, 1996; Whitlock et al., 2000). Detection of the infected, nonshedding animals will, therefore, rely either on progression of the disease to the shedding stage or through measurement of an immune response. Use of tests such as interferon- $\gamma$ assays to demonstrate cell-mediated immunity is an option (McDonald et al., 1999), but these tests are not yet widely used. ELISA for antibody demonstration are, on the other hand, routinely used in some countries, but the drawback of these tests is that the sensitivity is not optimal in cows until their 2nd or 3rd parity if the ELISA is interpreted as a point estimate for the individual cow due to the chronic nature of the disease. Studies describing the dynamics of the ELISA for naturally infected cows in different stages of lactation have not been described. The dynamics are essential when interpreting the ELISA response as a binary outcome, which is the regular interpretation of ELISA used for infec- 
tious diseases. If changes do occur across lactation because the infected cow concentrates antibodies at certain times in lactation, this information can be used to draw inferences on the infection status of a cow at an earlier stage in her life, thus increasing the sensitivity of the diagnostic test. Changes are more easily demonstrated with continuous outcomes. Because not all cows infected with $M$. avium subsp. paratuberculosis will reach the shedding stage of infection (Whitlock and Buergelt, 1996), but can still contribute to a positive infection status of a herd, it is desirable to be able to identify these infected cows. However, the immune response of some of these cows is primarily cell-mediated (Stabel, 2000). Therefore, to identify these cows based on antibodies, the course of the antibody levels during lactation needs to be known. Uninfected cows are expected to have no antibodies, and infected cows are expected to show different patterns depending on whether they will shed bacteria or not.

The first objective of the present study was to determine how the antibody response to infection with $M$. avium subsp. paratuberculosis changes with time across lactation and within parity. The second objective was to use the ELISA absorbance level and the dynamics of the antibodies to identify paratuberculosis infection in dairy cattle.

\section{MATERIALS AND METHODS}

\section{Herds, Animals, and Observations}

Seven herds in which M. avium subsp. paratuberculosis had been cultured from fecal samples within 6 mo before the start of the study period were selected for the study. The herd sizes, on average, were from 68 to 121 (median: 86 cows). Samples from 854 cows were collected from the routine milk production scheme in which samples were collected 11 times per year. The samples were collected from the herds from January 2000 to January 2001. Observations from cows collected at more than 280 DIM (= $40 \mathrm{wk}$ ) were excluded from data analyses. The composition of animals by breed subsequent to this data editing was as follows: 1 Danish Red-and-White, 255 Danish Jersey, 480 Danish Holsteins, 20 Red Danish, and 61 cross-breeds. Sampling included all cows lactating on the day of sampling; hence, the number of samples per cow differed: 1 from 88 cows, 2 from 59 cows, 3 from 54 cows, 4 from 79 cows, 5 from 101 cows, 6 from 114 cows, 7 from 160 cows, 8 from 121 cows, 9 from 32 cows, and 10 samples from 4 cows giving a total of 4289 samples from 812 cows. There were 427 cows in first (1980 samples), 245 in second (963 samples), 157 cows in third (655 samples), 87 cows in fourth (388 samples), and 80 cows (383 samples) in fifth parity (the number of cows does not add up to 812 as some cows shifted parity during the study). In addition, fecal samples were collected from all cows in the herds at three time points in the study. Thus, sampling was done once for 326 cows, twice for 252 cows, and three times for the remaining 334 cows, depending on their entrance into the study and exit due to culling.

\section{Diagnostic Tests}

All samples collected were treated with bronopol (a preservative) immediately after sampling, and upon arrival at the laboratory of the Danish Dairy Board, the samples were centrifuged and the fat fraction was removed. The skim milk was then frozen for later testing in an indirect ELISA.

The ELISA was performed as described elsewhere (Nielsen et al., 2001) except for the following modifications: The milk samples were diluted 1:2 in Mycobacterium phlei with no further dilution. Antigen was used at a concentration of $1.00 \mu \mathrm{g} / \mathrm{ml}$, and the conjugate was diluted 1:2000 in phosphate-buffered saline. Each sample was tested in duplicate, and to each ELISA plate was added a standard milk sample in four wells. The ELISA results were then read as optical density (OD) values for each sample. Correction for changes in ELISA readings due to laboratory factors was done as described in the section on statistical analyses (below).

Fecal culture (FC) was done on Löwenstein-Jensen medium subsequent to preincubation and decontamination using $\mathrm{NaOH}$ and oxalic acid, followed by neomycin and amphotericin B. Growth on the culture medium was determined 5, 8, and 12 wk after incubation. Further details on the diagnostic tests and their evaluations are described elsewhere (Nielsen et al., 2002). Cows were classified as FC-positive if a single colony was detected on the medium for any sample.

\section{Statistical Analyses}

To determine changes in the ELISA readings, the OD values were analyzed on a continuous scale and not dichotomized, as is usual. Linear regression analysis was performed using the mixed model procedure in SAS according to the guidelines given by Singer (1998) and Littell et al. (1996).

First, descriptive statistics were performed. For this purpose, the OD values were grouped into four categories by three arbitrarily selected cut-off values: $\mathrm{OD}=$ $0.100, \mathrm{OD}=0.250$, and $\mathrm{OD}=1.000$. The mean of the OD values for various groups was also evaluated. Some of the independent variables were also categorized initially into five parity groups $(1=1,2=2,3=3,4=4$, and $5=\geq 5$ ), 22 groups for stage of lactation (in groups 
of 2 -wk intervals), and three groups of age at 1 st calving $(1=20-25 \mathrm{mo}, 2=26-30 \mathrm{mo}, 3=>30 \mathrm{mo})$.

Based on the descriptive statistics, two categories were collapsed into fewer groups: Two breed groups $\left(\mathrm{B}_{p}\right)$ were created, $p=1$ for Danish Holsteins and crossbreeds, and $p=0$ for Danish Jerseys, Danish Red-andWhite, and Red Danish. Three parity groups $\left(\mathrm{P}_{j}\right)$ were created: $j=1$ for parity $1, j=2$ for parity 2 , and $j=3$ for parity $\geq 3$. In subsequent multivariable analyses, stage of lactation was evaluated both as a categorical variable and as a continuous variable. The remainder of the variables was evaluated as given here.

The dilution effect of milk production volume on antibody concentration was also considered, as it has been reported that the IgG $\mathrm{I}_{1}$ concentration can be influenced by the volume of milk (Pritchett et al., 1991). The predicted milk production $\left(\mathrm{PM}_{i j}\right)$ on the $i^{\text {th }}$ day in milk $\left(\mathrm{D}_{i}\right)$ for cows in the $j^{\text {th }}$ parity $\left(\mathrm{P}_{j}\right)$ was calculated using the formula:

$$
\mathrm{PM}_{i j}=b_{0}+b_{1} \times \frac{\mathrm{D}_{i}-60}{245}+b_{2} \times \frac{\mathrm{D}_{i}-60}{245}+\mathrm{P}_{j},
$$

where $b_{0}$ is the estimated mean milk production at $\mathrm{d}$ 60 in milk, $b_{1}$ is the coefficient for the change in milk production from d 1 to $59 \mathrm{in} \mathrm{milk,} \mathrm{and} b_{2}$ is the coefficient for the change in milk from d 60 to 305 . The difference between predicted and observed milk production $(\mathrm{kg})$ on the day of sampling was then calculated by subtracting the actual milk production from the predicted. This piece-wise linear model is a modification of the Wilmink-function (Wilmink, 1987). The parameters were estimated based on the present material.

Subsequent to the descriptive analyses and recategorizing of the independent variables, univariable analyses were carried out. Because ELISA results usually have a skewed distribution, a log transformation of these was performed, thereby normalizing them reasonably. The resulting response variable was designated $\ln (\mathrm{OD})$. No variable selection was performed at this step of the statistical analyses.

The ELISA response is a composition of background coloring due to various laboratory factors and the concentration of antibodies. A noninfected animal would be expected to have a concentration of antibodies equal to zero, but laboratory factors could induce a coloring that gives an OD reading greater than zero. To diminish potential effects of background coloring, the 10th percentile was calculated for each ELISA plate and subtracted from the mean OD reading for each sample. To correct for additional laboratory effects and clustering effects, a fixed effect of the combination of ELISA-plate (E), laboratory test-day (T), herd (H), and breed group (B) was included in the model (as an interaction be- tween the four, $\left.\mathrm{E}^{*} \mathrm{~T}^{*} \mathrm{H}^{*} \mathrm{~B}\right)$. To correct for additional cow-time-factors not related to DIM and parity, age at first calving (A) was included.

The interaction between DIM and parity was highly significant. Hence, DIM was nested in parity. The time shortly after calving has a higher likelihood and the mid-lactation lower likelihood, of being antibody positive. Therefore, DIM was included in the model allowing piecewise linear combinations for DIM and modeled with a random coefficient for each cow (Littell et al., 1996). Day 60 was selected as the pivotal point in this 'hockey-stick' model for the following reasons: 1) exploratory analyses indicated that the lowest OD values generally occurred around this stage of lactation, and 2) this cut-off allowed more test days in a greater number of the study subjects. The mean effects of parity and of DIM nested in parity were included in the model as fixed effects, and three components for the random effects of DIM were included for each cow: a random intercept, a random slope prior to $\mathrm{d} 60$, and a random slope after d 60; that is, intercepts and slopes could differ between cows within lactation. Variation was allowed to be different for each parity, and the random effects were modeled using the unstructured type of covariance matrix for the random coefficients, with the individual cows as subjects.

The full multivariable 'base model' was:

$$
\begin{aligned}
& \mathrm{Y}_{i j k l m n p q r s t}=\mathrm{U}+\mathrm{P}_{j}+b_{1} \times \text { Dun60 } 0_{i j k}+b_{2} \\
& \times \mathrm{D} 60_{i j k}+\mathrm{FC}_{l}+\mathrm{A}_{m}+b_{3} \times \mathrm{MD}_{i j}+\mathrm{H}_{n} \times \mathrm{B}_{p} \\
& \times \mathrm{E}_{q} \times \mathrm{T}_{r}+\mathrm{C}_{s t}+\mathrm{e}_{i j k l m n p q r s t}
\end{aligned}
$$

where

$\mathrm{Y}_{\text {ijklmnpqrst }}=$ the transformed ELISA result, $\ln (\mathrm{OD})$ for the $s$ th sample from the $t$ th cow in the $n$th herd producing MD kg deviation of milk from expected on the $i$ th DIM in the $j$ th parity for cows in the $m$ th group of age at 1 st calving if the cows belonged to the $p$ th breed group and where tested in the $q$ th ELISA-plate on the $r$ th test day in the laboratory;

$\mathrm{U}=$ the mean of the ELISA at the intercept;

$\mathrm{P}_{j}=$ the fixed effect of the $j$ th parity;

Dun60 $0_{i j k}=$ the linear effect of the $i$ th DIM in parity $j$, where $i=1-59$ and $j=1,2, \geq 3$ (see above) and $k=1$, when Dun60 is included as a fixed effect, and $k=2$ when included as a random effect;

$\mathrm{D} 60_{i j k}=$ the effect of the $i$ th DIM in parity $j$, where $i=1-280$ and $j=1,2, \geq 3$ (see above), and $k=1$, when D60 is included as a fixed effect, and $k=2$ when included as a ran- 


$$
\begin{aligned}
& \text { dom effect; } \\
\mathrm{FC}_{l}= & \text { the fixed effect of the } l \text { th result from fecal } \\
& \text { culture, } \mathrm{FC} ; \\
\mathrm{A}_{m}= & \text { the fixed effect of the } m \text { th group of age at } \\
& \text { 1st calving, A; } \\
\mathrm{MD}_{i j}= & \text { the fixed effect of the observed minus the } \\
& \text { expected milk production }(\mathrm{kg}) \text { of the cow } \\
& \text { on the } i \text { th day in lactation in parity } j ; \\
\mathrm{H}_{n}= & \text { the fixed effect of the } n \text {th herd, H; } \\
\mathrm{B}_{p}= & \text { the fixed effect of the } p \text { th breed group, B; } \\
\mathrm{E}_{q}= & \text { the fixed effect from the } \text { th ELISA-plate } \\
& \text { E; } \\
\mathrm{T}_{r}= & \text { the fixed effect from the } r \text { th ELISA test- } \\
& \text { day, T; } \\
\mathrm{C}_{s t}= & \text { the effect of the } s \text { th sample from the } t \text { th } \\
& \text { cow, C, included as a random effect with } \\
& \text { unstructured type of covariance between } \\
& \text { the } s \text { th and the } s+1 \text { st sample; } \\
b_{1}, b_{2}, \text { and }= & \text { the regression coefficients for Dun } 60_{i j k}, \\
b_{3} \quad & \text { D60 } 0_{i j k} \text {, and MD } \mathrm{MD}_{i j}, \text { respectively; } \\
e_{i j k l m n p r s t}= & \text { a random residual component normally } \\
& \text { distributed, } \mathrm{N}\left(0, \sigma_{e}^{2}\right)
\end{aligned}
$$$$
\text { and }\left(\begin{array}{c}
\mathbf{C}_{i s t(j)} \\
\operatorname{Dun60}_{i(j)} \\
\mathbf{D} 60_{i(j)}
\end{array}\right) \sim N\left[\left(\begin{array}{l}
0 \\
0 \\
0
\end{array}\right),\left(\begin{array}{lll}
\tau \mathbf{P}_{j 11} & \tau \mathbf{P}_{j 12} & \tau \mathbf{P}_{j 13} \\
\tau \mathbf{P}_{j 21} & \tau \mathbf{P}_{j 22} & \tau \mathbf{P}_{j 23} \\
\tau \mathbf{P}_{j 31} & \tau \mathbf{P}_{j 32} & \tau \mathbf{P}_{j 33}
\end{array}\right)\right]
$$

for each parity group $j=1, j=2$, and $j=3$.

In the above 'base model', 2nd and 3rd order interactions were evaluated for the fixed effects. Given that interaction terms were significant, DIM $\left(\mathrm{D}_{i j k}\right)$ was nested in parity $\left(\mathrm{P}_{j}\right)$. The combined effect of breed group $\left(\mathrm{B}_{p}\right)$, herd $\left(\mathrm{H}_{i}\right)$, ELISA-plate $\left(\mathrm{E}_{q}\right)$, and ELISA test-day $\left(\mathrm{T}_{r}\right)$ was only evaluated as an interaction.

The initial multivariable analyses performed ignored the fact that only 'infected animals' would have variation in their antibody concentrations. As a matter of fact, the true infection status was unknown. Obviously, animals with positive FC could be considered infected, while those with negative FC are not necessarily free of infection, as the sensitivity of the FC varies in the range of approximately 20 to $70 \%$, depending on the progression of the disease (Nielsen et al., 2002). As the contribution from the background coloring and the antibodies cannot objectively be separated, and as the true infection status of none of the study cows was known, it was attempted to separate 'uninfected animals' from 'infected animals' based on inter animal comparison. Rather than setting a fixed diagnostic parameter as a gold standard, the solutions from the random coefficients models were used to cross-classify animals into cows with antibody levels that were significantly higher $(P<0.05)$ than the mean (intercept) or those with changes in antibody levels (the slope of the coefficients Dun60 and D60 were significantly different from 0). These cross-classifications were done separately for FCpositive and FC-negative cows.

Predicted OD values at different time points were calculated for each cow using the model above, including all fixed effects and including the parameters of the within-cow antibody response profile (repeated measurements) as random effects with unstructured covariance.

All multivariable analyses were carried out using manual backward elimination at a 95\% level of significance based on the likelihood ratio test, and by assessing model fit with Akaike's Information Criterion and the Schwarz Bayesian Information Criterion. Plots of residuals $\left(\mathrm{e}_{i j k l m n p s t}\right)$ and predicted values were performed to evaluate heteroscedasticity, and it was evaluated whether the residuals had a normal distribution as assumed in the 'base model'.

\section{RESULTS}

The mean OD values from the ELISA for all milk samples collected prior to $\mathrm{d} 281$ from all cows are given in Table 1 by parity and stage of lactation, separated for FC-positive and FC-negative cows. Fitting a regression model based on these 4289 observations from 812 cows with unknown true infection status yielded a regression model including the terms represented in equation 1 (Materials and Methods). None of the 2nd and 3rd order interaction terms were found significant except the interaction between the terms of DIM and parity. Hence, they were nested in parity. The variance and covariances were different for different parities $(P<0.0001)$; therefore, difference in covariance for each of the parity groups was allowed in the model. The model converged in seven iterations without any indications of estimation problems. The average predicted piece-wise linear antibody responses for each of the parity groups and for FC-positive and FC-negative cows are given in Figure 1. The predicted piece-wise linear antibody responses for each FC-positive and FC-negative cow by parity are given in Figure 2. Some cows showed marked instability, i.e., one or both of the slopes of the piece-wise linear plot were different from 0 , some cows showed high levels of antibodies (high OD, higher than the mean/intercept), and some cows showed both. The distribution of cows showing high level, instability, or either is shown in Table 2. The apparent prevalence of infection based on FC was $8 \%$ (66/812), while based on high OD level it was $12 \%$ (98/812), and based on high OD level or instability it was $15 \%(125 / 812)$. 
Table 1. Mean ELISA values for 4289 milk samples from 746 fecal culture-negative and 66 fecal culturepositive cows from 7 Danish dairy herds by parity and stage of lactation.

\begin{tabular}{|c|c|c|c|c|c|c|}
\hline \multirow{3}{*}{$\begin{array}{l}\text { Stage of } \\
\text { lactation } \\
\text { (wk) }\end{array}$} & \multicolumn{3}{|c|}{$\begin{array}{l}\text { Mean OD value of samples }(\mathrm{n}=3966) \text { from } \\
746 \text { fecal culture-negative cows }{ }^{1}\end{array}$} & \multicolumn{3}{|c|}{$\begin{array}{c}\text { Mean OD value of samples }(\mathrm{n}=323) \text { from } 66 \\
\text { fecal culture-positive cows }{ }^{1}\end{array}$} \\
\hline & \multicolumn{3}{|c|}{ Parity } & \multicolumn{3}{|c|}{ Parity } \\
\hline & 1 & 2 & $\geq 3$ & 1 & 2 & $\geq 3$ \\
\hline $1-2$ & $0.184(69)$ & $0.246(34)$ & $0.324(52)$ & $0.124(2)$ & $0.261 \quad(3)$ & $0.212(4)$ \\
\hline $3-4$ & $0.145(104)$ & $0.166(60)$ & $0.182(76)$ & $0.332(4)$ & 0.606 (14) & $0.755(6)$ \\
\hline $5-6$ & 0.113 & $0.175(51)$ & $0.194 \quad(83)$ & 0.196 & $0.074(3)$ & $0.643(8)$ \\
\hline $7-8$ & $0.113(98)$ & $0.153(38)$ & $0.219(66)$ & $0.090(2)$ & $0.734(3)$ & $0.673(8)$ \\
\hline $9-10$ & 0.116 & $0.159(44)$ & $0.176(75)$ & $0.233(5)$ & $0.331(8)$ & 0.453 \\
\hline $11-12$ & 0.118 & $0.155(51)$ & $0.169(73)$ & $0.117(7)$ & $0.241(3)$ & $0.378(6)$ \\
\hline $13-14$ & 0.116 & $0.152(34)$ & $0.219(54)$ & $0.147(2)$ & $0.260(2)$ & $0.501(9)$ \\
\hline $15-16$ & $0.126(98)$ & $0.135(50)$ & $0.211(71)$ & $0.124(8)$ & $0.084(4)$ & 0.527 (4) \\
\hline $17-18$ & $0.129(88)$ & $0.176(37)$ & $0.201 \quad(59)$ & $0.236(7)$ & $0.140(2)$ & $0.350(9)$ \\
\hline $19-20$ & $0.141(93)$ & $0.143(36)$ & $0.245(60)$ & $0.200(5)$ & $0.276(2)$ & $0.421(7)$ \\
\hline $21-22$ & $0.125(101)$ & $0.175(54)$ & $0.214(73)$ & 0.234 (11) & $0.207(5)$ & 0.963 \\
\hline $23-24$ & $0.121(71)$ & $0.128(33)$ & $0.217(61)$ & $0.141(3)$ & $0.485(4)$ & $0.359(7)$ \\
\hline $25-26$ & $0.162(88)$ & 0.148 (44) & $0.214(73)$ & $0.472(9)$ & 0.359 (4) & $0.845(7)$ \\
\hline $27-28$ & $0.119(88)$ & $0.202(45)$ & $0.252(57)$ & $0.249(6)$ & 0.211 & $0.461(6)$ \\
\hline $29-30$ & $0.190(83)$ & $0.140(42)$ & $0.230(64)$ & $0.488(8)$ & $0.651(3)$ & $0.464(6)$ \\
\hline $31-32$ & $0.107(82)$ & $0.220(51)$ & $0.243(73)$ & $0.729(6)$ & 0.528 & $0.542(9)$ \\
\hline $33-34$ & $0.142(80)$ & $0.173(37)$ & $0.198(50)$ & $0.453(9)$ & $0.541(2)$ & $0.758(6)$ \\
\hline $35-36$ & $0.141 \quad(82)$ & $0.224(49)$ & $0.198(69)$ & $0.347(6)$ & $0.602(2)$ & 0.718 \\
\hline $37-38$ & $0.172(73)$ & $0.201(46)$ & $0.253(66)$ & $0.431(8)$ & $0.606(4)$ & $0.870(7)$ \\
\hline $39-40$ & $0.163(72)$ & 0.186 & $0.241(48)$ & $0.491(10)$ & $0.922(2)$ & 0.443 \\
\hline Total & 0.136 & 0.174 & 0.217 (1303) & $0.328(122)$ & $0.422(78)$ & $0.565(123)$ \\
\hline
\end{tabular}

${ }^{1}$ The number of observations per group is given in parentheses.

\section{DISCUSSION}

The diagnosis of paratuberculosis in infected herds is challenging. Initially, animals actively shedding the mycobacteria are often detected if a sound FC strategy is used. Later in the process, when shedding cows are removed from the herd, infected animals are likely to

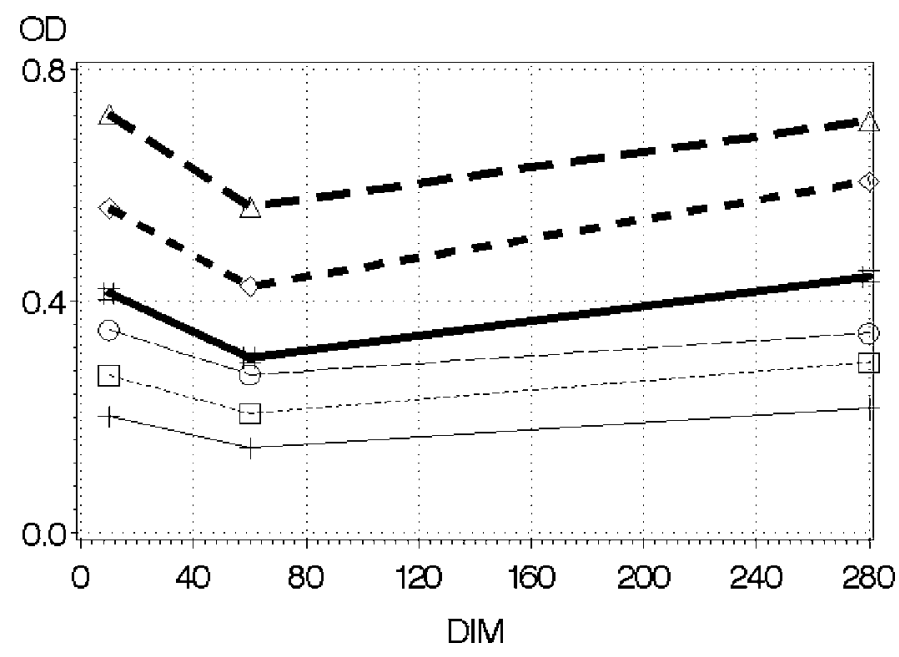

Figure 1. The average predicted piece-wise linear profiles of the antibody responses plotted for 746 FC-negative cows (lower three thin 'hockey-sticks') and 66 FC-positive cows (top three fat 'hockeysticks') for each of the parity groups: parity 1 (\# and + ), parity 2 ( $\square$ and $\diamond)$, and parity $\geq 3(\triangle$ and $\bigcirc)$. be present, but FC cannot detect these animals if they are not actively shedding (Nielsen et al., 2002). The problems of detecting latently infected cows could be due to the intermittent shedding of mycobacteria in some cows, the lack of shedding from cows with the infection under control, and the lack of an agent detection method providing almost $100 \%$ sensitivity. If the mycobacteria cannot be detected using an agent detection method such as the FC, an alternative is the use of immunological tests such as the ELISA. A few months post infection, some infected animals will already have an active immune response (Lepper et al., 1989; McDonald et al., 1999) with presence of antibodies, but it is likely that many animals will not develop a strong immune response while the dominating cellmediated immune response in early infection stages of paratuberculosis is keeping the antibody production in check. Utilizing the cows' abilities to concentrate these antibodies in early lactation in connection with colostrum formation provides a diagnostic potential that has yet to be realized. In this study, it was demonstrated that the antibody levels in nonshedding individual cows actually do change quite a bit at the beginning of lactation even for first-lactation cows that normally have a much lower antibody level (Figure 1). However, the average plots include two groups of cows that do not have changes in antibody level: the truly noninfected cows and the cows already at the high level. Hence, the 


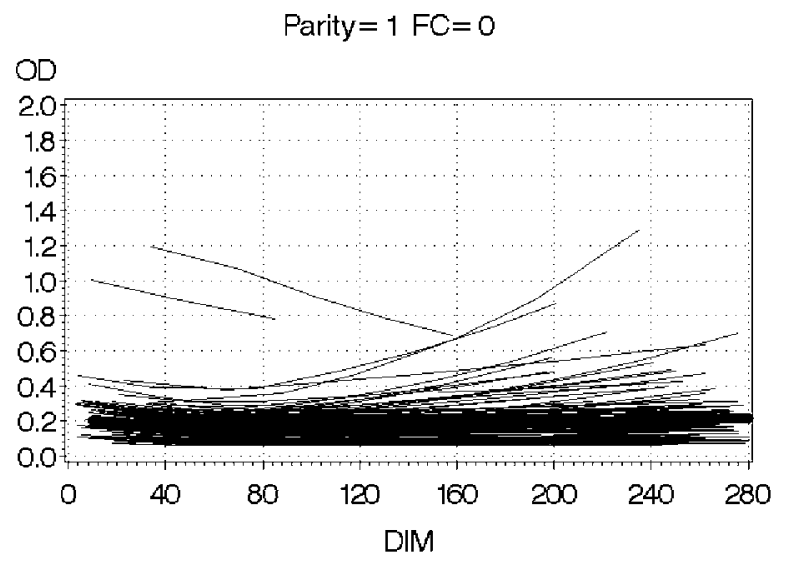

Parity $=2 \quad F C=0$

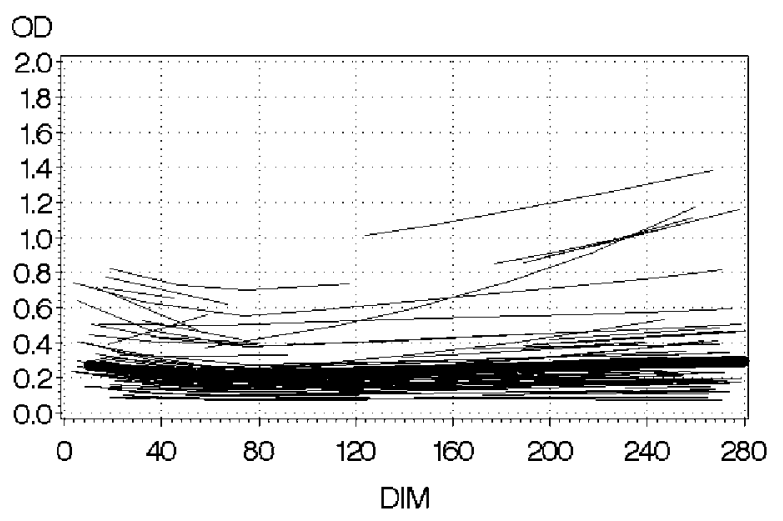

Parity $=3 \quad F C=0$

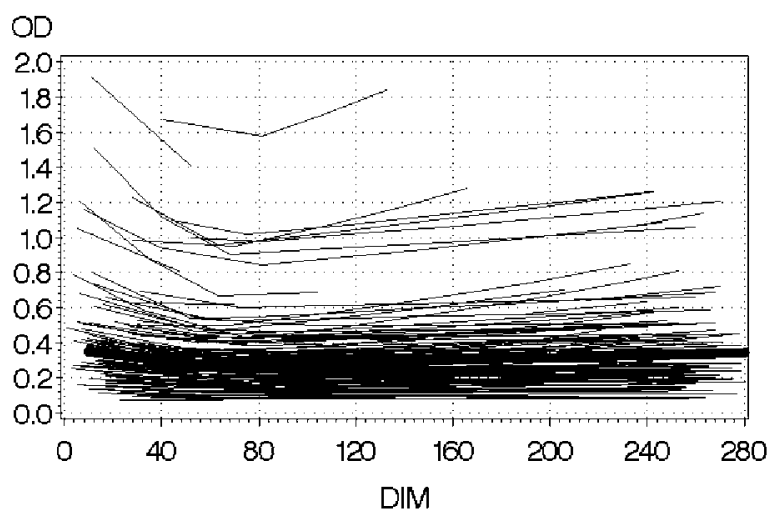

Parity $=1 \mathrm{FC}=1$

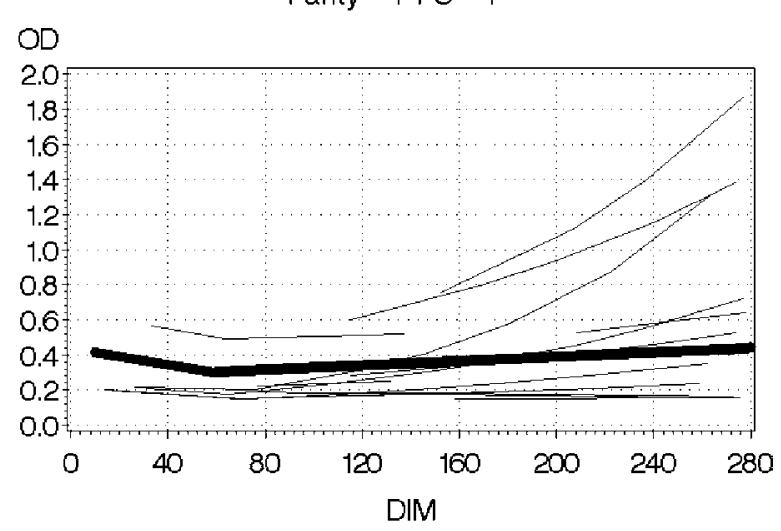

Parity $=2 \mathrm{FC}=1$

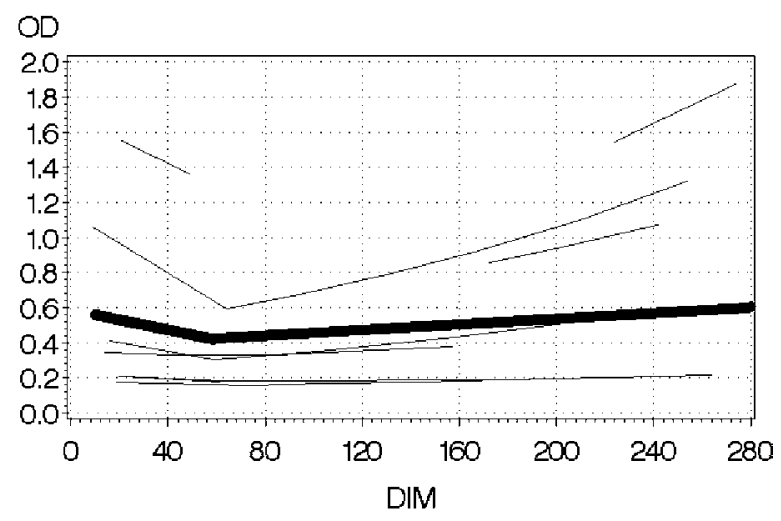

Parity $=3 \quad F C=1$

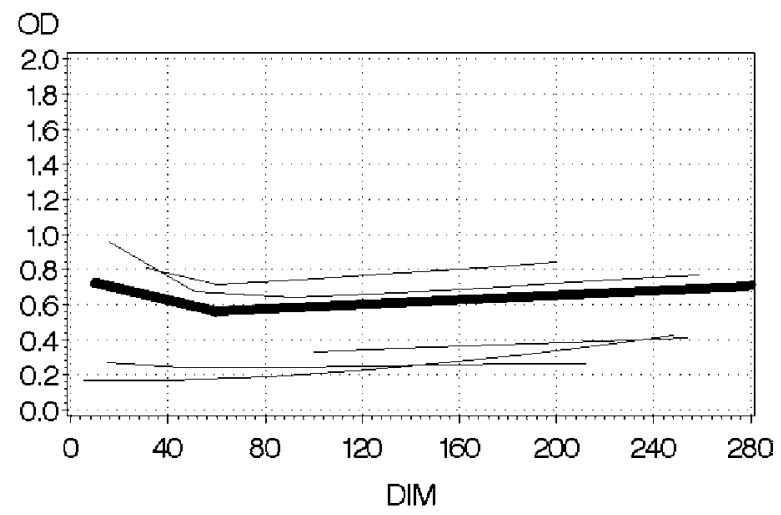

Figure 2. The predicted piece-wise linear profiles of the antibody responses plotted for each of $746 \mathrm{FC}-$ negative cows $(\mathrm{FC}=0)$ and 66 FC-positive cows $(\mathrm{FC}=1)$ by parity groups 1,2 , and parity $\geq 3$. Each of the thin lines represents one cow nested in parity. The fat lines are the average predicted lines from Figure 1.

solutions by cow from the random coefficients models (Figure 2) show a much more differentiated view of the population, and, just by visual inspection, some cows have antibody patterns much different from those of others either because the antibody level is higher or because there is instability in the immune response. For instance, 1st-parity FC-negative cows show a development very similar to that of most FC-positive cows.
It could be speculated that the FC-negative cows with changes in the antibody response are paratuberculosis infected, though this has not been proven. But it raises the question to a decision maker whether it is wise to keep unstable cows in the herd. They are at risk of becoming shedders, thus infecting other cows. But this might never happen. At the end of an eradication program in a herd, use of antibody dynamics can be used 
Table 2. Distribution of 746 fecal culture-negative and 66 fecal culture-positive cows from seven Danish dairy herds according to antibody level and stability. ${ }^{1}$

\begin{tabular}{llrrr}
\hline & & \multicolumn{3}{c}{ Fecalculture } \\
\cline { 3 - 5 } Immune status & & + & \multicolumn{3}{c}{ Total } \\
\hline Antibody level & High & 14 & 84 & 98 \\
\multirow{3}{*}{ Stability } & Low & 52 & 662 & 714 \\
& Change & 7 & 35 & 42 \\
Antibody level and stability & No change & 59 & 711 & 770 \\
& High or change & 20 & 105 & 125 \\
& Low and no change & 46 & 641 & 687 \\
\hline
\end{tabular}

${ }^{1}$ Change was defined as one or more slopes different from $0(P<0.05)$, and high level was defined as an intercept higher than the overall intercept $(P<0.05)$. These estimates are based on a model including Herd*Breed-group*ELISA-plate*ELISA-test day as a combined fixed effect, age-at-1st calving, parity, piecewise linear estimates of DIM, milk dilution effect and fecal culture as other fixed effects and the effect of each cow as a random coefficient, and a piece-wise linear effect of DIM for separate parity groups.

to identify the last infected nonshedding cows in the herd. Final proof that the antibodies are really directed to $M$. avium subsp. paratuberculosis infection will often not be available, and such proof could be impossible to retrieve.

According to the plots in Figure 2, the antibody level is much higher at the beginning and the end of lactation. There is quite a difference between individual cows in the antibody pattern, and this dynamic may be utilized. Optimal sampling times for single samples could be the beginning or end of lactation. But no matter which sampling time is used, corrections for the sampling time should be included in the interpretation of the ELISA result. Repeated samplings (with a minimum of two samples) to determine stability would have a higher diagnostic value and, therefore, aid in a specific diagnosis. Repeated samples provide a gain in sensitivity without a loss in specificity. This loss in specificity is normally a problem when evaluating diagnostic tests with a continuous response but not here because within-cow the specificity can be regarded as constant.

In general, the antibody responses in $\mathrm{FC}$-positive cows are much higher than in FC-negative cows. Still, some FC-positive cows do not show any antibody production at all. Therefore, combining ELISA and FC seems to be appropriate. Interpreting the diagnostic tests in parallel (FC, ELISA level, and ELISA stability) provides a higher combined sensitivity of the tests, which can be desirable in some situations where the prevalence is low. In the present study, 125 cows were identified as positive based on either ELISA level or ELISA stability, and an additional 34 were FC-positive, i.e., a total of 159 of the cows were test-positive in either test, suggesting that almost $20 \%$ of the cows were infected (Table 2). This is not unreasonable, considering that the herds were selected as the ones with most antibody reactors (at a fixed cut-off for the ELISA in which the sensitivity was estimated to be 0.56 and the specificity 0.86 ) among more than 100 herds based on the ELISA results from a single screening. However, in these herds, it would still be advisable to follow a culling policy based on the FC-positive animals first, and in later stages of a control program, include ELISA reactions. The antibody response could also be used to predict shedding of the mycobacteria, but the design of the present study does not support changes in antibody concentration as a predictor of fecal shedding, although it does indicate that such changes could be used. To study this, concomitant sampling of milk and fecal samples over a longer time period would be necessary with subsequent event time analyses performed on the data.

\section{CONCLUSIONS}

The present study demonstrated that the concentration of antibodies to paratuberculosis changed greatly across lactation for some cows, nonshedding (FC-negative) cows as well as shedding cows (FC-positive). These changes were used to differentiate cows that were infected from cows that were not infected with $M$. avium subsp. paratuberculosis; thus, a tentative diagnosis could be made if other tests fail to identify the remaining cows in a herd. These changes were most prominent in 1st- and 2nd-parity cows, but these were also the cows in which low levels of antibodies were generally expected due to the shorter incubation period of these cows in relation to the assumed calfhood infection. If used with sequential testing with repeated samplings during a lactation, changes would demonstrate antibody-positive cows and perhaps even infected cows.

\section{ACKNOWLEDGMENTS}

The Danish Dairy Board and the Kongeåproject are thanked for their financial support of the present study. 


\section{REFERENCES}

Chiodini, R. J., H. J. van Kruiningen, and R. S. Merkal. 1984. Ruminant paratuberculosis (Johne's disease): The current status and future prospects. Cornell Vet. 74:218-262.

Larsen, A. B., R. S. Merkal, and R. C. Cutlip. 1975. Age of cattle as related to resistance to infection with Mycobacterium paratuberculosis. Am. J. Vet. Res. 36:255-257.

Lepper, A. W. D., C. R. Wilks, M. Kotiw, J. T. Whitehead, and K. S. Swart. 1989. Sequential bacteriological observation in relation to cell-mediated and humoral antibody responses of cattle infected with Mycobacterium paratuberculosis and maintained on normal or high iron intake. Aust. Vet. J. 66:50-55.

Littell, R. C., G. A. Milliken, W. W. Stroup, and R. D. Wolfinger. 1996. SAS ${ }^{\circledR}$ system for mixed models. SAS Inst., Inc., Cary, NC.

McDonald, W. L., S. E. Ridge, A. F. Hope, and R. J. Condron. 1999. Evaluation of diagnostic tests for Johne's disease in young cattle. Aust. Vet. J. 77:113-119.

Muskens J., H. W. Barkema, E. Russchen, K. van Maanen, Y. H. Schukken, and D. Bakker. 2000. Prevalence and regional distribution of paratuberculosis in dairy herds in the Netherlands. Vet. Microbiol. 77:253-261.

NAHMS. 1997. Johne's disease on US dairy operations. USDA:APHIS:VS, CEAH, National Animal Health Monitoring System, Fort Collins, CO, USA, No. N245.1097.

Nielsen, S. S., S. M. Thamsborg, H. Houe, and V. Bitsch. 2000. Bulktank milk ELISA antibodies for estimating the prevalence of paratuberculosis in Danish dairy herds. Prev. Vet. Med. 44:1-7 (with Corrigendum in Prev. Vet. Med. 46:297).

Nielsen, S. S., H. Houe, S. M. Thamsborg, and V. Bitsch. 2001. Comparison of two ELISAs for serological diagnosis of paratuberculosis (Johne's disease) using different subspecies strains of Mycobacterium avium. J. Vet. Diagn. Invest. 13:164-166.
Nielsen, S. S., C. Grønbæk, J. F. Agger, and H. Houe. 2002. Maximumlikelihood estimation of sensitivity and specificity of ELISA and faecal culture for diagnosis of paratuberculosis. Prev. Vet. Med. 53:191-202.

Pritchett, L. C., C. C. Gay, T. E. Besser, and D. D. Hancock. 1991. Management and production factors influencing immunoglobulin G1 concentration in colostrum from Holstein cows. J. Dairy Sci. 74:2336-2341.

Rankin, J. M. 1962. The experimental infection of cattle with Mycobacterium paratuberculosis. IV. Adult cattle maintained in an infectious environment. J. Comp. Pathol. 72:113-117.

Seitz, S. E., L. E. Heider, W. D. Hueston, S. Bech-Nielsen, D. M. Rings, and L. Spangler. 1989. Bovine fetal infection with Mycobacterium paratuberculosis. J. Am. Vet. Med. Assoc. 194:1423-1426.

Singer, J. D. 1998. Using SAS PROC MIXED to fit multilevel models, hierachical models, and individual growth models. J. Educational Behavioral Statistics 24:323-355.

Stabel, J. R. 2000. Transitions in immune responses to Mycobacterium paratuberculosis. Vet. Microbiol. 77:465-473.

Sweeney, R. W., R. H. Whitlock, and A. E. Rosenberger. 1992. Mycobacterium paratuberculosis isolated from fetuses of infected cows not manifesting signs of the disease. Am. J. Vet. Res. 53:477-480.

Wells, S. J., S. L. Ott, and A. H. Seitzinger. 1998. Key health issues for dairy cattle-new and old. J. Dairy Sci. 81:3029-3035.

Whitlock, R. H., and C. Buergelt. 1996. Preclinical and clinical manifestations of paratuberculosis (including pathology). Vet. Clin. North Am. Food Anim. Pract. 12:345-356.

Whitlock, R. H., S. J. Wells, R. W. Sweeney, and J. van Tiem. 2000. ELISA and fecal culture for paratuberculosis (Johne's disease): sensitivity and specificity of each method. Vet. Microbiol. 77:387-398.

Wilmink, J. B. M. 1987. Adjustment of test-day milk, fat, and protein yield for age, season, and stage of lactation. Livest. Prod. Sci. $16: 335-348$ 